\title{
Evaluating the cleaning performance of rectangular slot nozzle and diffuser
}

\author{
Haixia Li*, Zhendong Hao, Qi Zhang \\ School of Mechanical and Power Engineering, Henan Polytechnic University, Jiaozuo 454000, China
}

Corresponding Author Email: lihx@hpu.edu.cn

https://doi.org/10.18280/ijht.360113

Received: 18 September 2017

Accepted: 22 December 2017

\section{Keywords:}

rectangular slot nozzle, diffuser, pulse jet cleaning, computational fluid dynamics $(C F D)$

\begin{abstract}
This paper aims to disclose how the pulse gas performance is affected by the configurations of the rectangular slot nozzle and the cake-pie type diffuser. To this end, the pulse gas performance of the nozzle was evaluated through several simulations based on the computational fluid dynamics (CFD). Specifically, the author briefly introduced the nozzle and the diffuser, the modelling and meshing operations, the governing equations of the CFD, as well as the numerical plan and solution convergence. Then, the simulation model was validated by comparing the simulated results with experimental data. Focusing on the flow field around the nozzle, the effects of nozzle configuration, diffuser configuration and nozzlediffuser distance on pulse gas cleaning were discussed in great details. Through the discussion, the author drew the following conclusions: Obvious shocking waves were observed near the nozzle outlet. The nozzle outlet height has a greater impact on pulse jet cleaning than other nozzle configuration parameters (e.g. nozzle outlet width and nozzle extension width), while the diffuser height has a greater impact on pulse jet cleaning than other diffuser configuration parameters (e.g. diffuser inlet width and inlet-throat distance). The primary, secondary and total masses increased with the height and width of nozzle outlet; as the nozzle extension width grew, the secondary and total masses also increased, but the primary mass stayed the same. The entrainment ratio is negatively correlated with the height and width of nozzle outlet, and positively with nozzle extension width. The primary mass had nothing to do with the diffuser configuration, while the secondary mass, the total mass and the entrainment ratio increased with the diffuser height, the diffuser width and the nozzle-diffuser distance. The research findings provide a valuable theoretical guidance for pulse jet cleaning system of filters.
\end{abstract}

\section{INTRODUCTION}

Recent decades have witnessed the extensive application of filtration in many industries $[1,2]$. However, the actual filtering effect is still constrained by the removal of dust cake [3 5]. To achieve effective removal, one of the viable options is to integrate pulse cleaning into the filtration technology. The most popular, feasible solution is the reverse pulse jet cleaning system [6]. The purpose of this system is to prevent patch cleaning through applying sufficient, uniform pressure along the filter length. For this purpose, a high-speed primary jet is injected via a nozzle into the filter cavity to form the primary flow of the pulse gas. This flow creates a low-pressure zone around the nozzle, generates the suction momentum of the surrounding fluid, and induces a secondary gas flow $[7,8]$.

The secondary gas flow has some positive effects on the operating effect of the filter. First, the additional gas injected into the filter cavity enhances the cleaning effect of the filter element, and increases the reverse momentum to remove the dust cake from the filter element. Second, the hot gas entrained in the secondary gas flow reduces the thermal shock during the injection of the cold pulse gas [9]. However, the secondary gas flow also has its downside: the suction effect is extended farther into the filter element, forming a negative pressure near the mouth of filter element. Therefore, it is very important to provide sufficient, uniform pulse pressure for pulse jet cleaning, creating the optimal working condition of the filter element. For the effective use of the pulse gas, the best method is to maximize the entrainment effect in the guiding area between the nozzle outlet and the diffuser mouth, so that negative pressure can form in the area and extend all the way up to the diffuser.

Many scholars have investigated the pulse jet cleaning system, aiming to optimize the entrainment effect and pressure distribution. For instance, Choi [2] compared the pulse jet cleaning effects of three basic types of conventional jet nozzles, and discovered that the convergent nozzle outperforms the straight nozzle thanks to its concentrating effect and highspeed jet gas. Following computational fluid dynamics (CFD), Gurulingam [10] conducted a numerical prediction of the impacts from independent variables (e.g. temperature, primary nozzle pressure and mass flow rate) to the entrainment ratio of the jet ejector. Lo [11] experimentally explored the effect of tank pressure on pressure distribution, and confirmed the strong correlation between the cleaning effect and the tank pressure. Weidman [12] discussed how pulse velocity, pulse duration, nozzle-filter distance affects the pulse jet cleaning effect, created a dimensionless operation number of pulsed jet flow cleaning processes to predict the cleaning effects of different filter media, and concluded that the cleaning effect increases with the decrease of pulse interval but does not correlate with pulse duration.

Yan [13] examined the static pressure distribution of the filter element during the pulse jet cleaning process with Laval nozzle and air diffuser, aiming to reduce the incidence of incomplete cleaning. Qian optimized the relationship between 
jet distance and nozzle diameter of a semi-industrial pulse jet cartridge filter [14]. Reference [15] reveals that the pulse gas creates an oscillating propagation of pressure intensity along the flow direction, forming repeating but decaying cycles of positive and negative values. The negative pressure pulls the gas from the surroundings, while the positive pressure pushes the gas outwards. To find the optimal condition of pulse jet cleaning, it is necessary to fully understand the oscillation phenomenon of the pulse gas.

The CFD analysis has been adopted to study the pulse jet cleaning system, because it is impossible to determine all the design values of solely based on experiments. For example, References [16, 17] employs the CFD software FLUENT a 2D microscale, seeking to grasp the nature of gas flow during pulse jet cleaning. Through CFD computation, Zhang et al. [18] discovered that the pressure oscillation generates at the nozzle outlet and propagates into the flow field of pulse jet cleaning. Besides, the CFD analyses are carried out to optimize the pressure of pulse gas tank [2,19, 20], mass flow rate [20], pulse duration $[9,16,20]$, nozzle-filter distance $[9,14,20]$, etc. With the aim to validate the FLUENT code, Ramy [21] contrasted the numerical simulation results of three different turbulence models with experimental data, and proved the applicability of the standard $\mathrm{k}-\varepsilon$ turbulence model to the simulation of nozzle and diffuser.

Nevertheless, the studies above mainly concentrate on tubular nozzle and single filter element. There are few reports on rectangular slot nozzle or group filter element. In the industrial field, the group filter element is often combined with a large tubular nozzle or a manifold of several small tubular nozzles, and installed onto the popular venturi type diffuser [22]. Despite their relatively good effects, the former combination consumes too much space, while the latter complicates the structure of the pulse jet cleaning system. By contrast, the diffuser, coupled with a rectangular slot nozzle, is compact and space-saving. This is because a hundred filter elements are embedded in a single diffuser [23] and the highpressure pulse gas is distributed above the elements as it propagates radially in the diffuser. To optimize the cleaning effect, it is necessary to maximize the amount of pulse gas entering the rectangular slot nozzle of the diffuser. Considering the limited measurement skills for experimental analysis and the numerous co-dependent influencing factors of the pulse effect, this paper introduces the CFD to evaluate how the pulse gas performance is affected by the configurations of the rectangular slot nozzle and the diffuser.

\section{METHODOLOGY}

\subsection{Nozzle and diffuser}

Figure 1 presents the rectangular slot nozzle and the diffuser. The $210 \mathrm{~mm}$-long venture-shape nozzle receives the pulse gas via an $80 \mathrm{~mm} \times 30 \mathrm{~mm}$ (with $\times$ height) rectangular mouth. The pulse gas converges in the first $60 \mathrm{~mm}$ segment between the mouth and the throat (60m wide), diverges in the next $90 \mathrm{~mm}$ segment, and converges again in the last $60 \mathrm{~mm}$ segment. Along the length direction, the nozzle height continues to shrink from the $30 \mathrm{~mm}$ at the mouth. For the standard nozzle, the nozzle outlet height $\left(\mathrm{h}_{\mathrm{n}}\right)$, nozzle outlet width $\left(\mathrm{w}_{\mathrm{n}}\right)$ and nozzle extension width $\left(\mathrm{e}_{\mathrm{n}}\right)$ were set to $30 \mathrm{~mm}, 60 \mathrm{~mm}$ and $90 \mathrm{~mm}$, respectively. The diffuser is pinched in the middle, making a carefully balanced, asymmetric hourglass shape. The length, throat width and outlet width of the diffuser were kept as constants, while the inlet width, height and the inlet-throat distance were adjusted to disclose the effect of the diffuser on the cleaning effect.

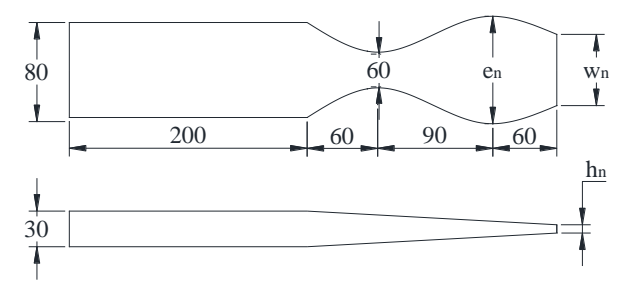

(a) Nozzle

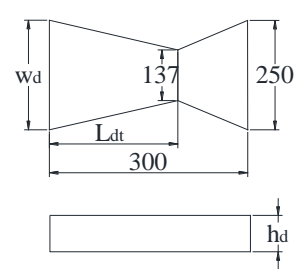

(b)Diffuser

Figure 1. Schematic of size and dimension of nozzle and diffuser

\subsection{Modelling and meshing}

This section discretizes the volume-averaged conservation equation of the filter element by the finite volume method [2426]. The specific steps include the geometric modelling and the grid meshing in GAMBIT. For accuracy, the structured quadrilateral units of the calculation domain were refined with strict size control. Then, the grid independent test was performed by refining the grids into the final pattern to check the convergence of the calculated results relative to spatial resolution [27]. Based on the static pressure along the nozzle axis, the test was carried out using four sets of grids. The test results are shown in Figure 2.

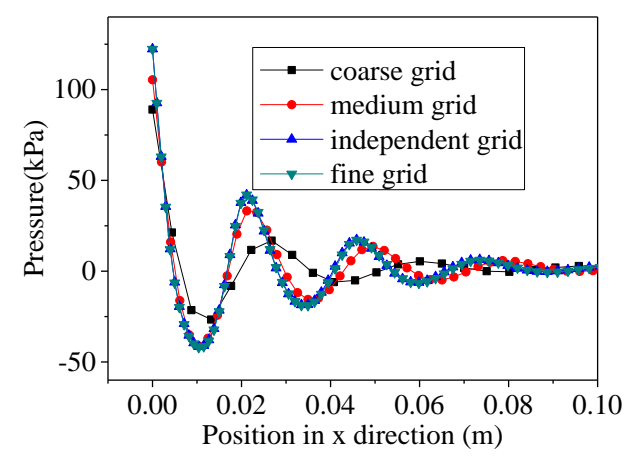

Figure 2. Grid independent examination

For the first set (coarse grid), the computing domain was meshed into 367,000 units. For the second set (medium grid), the computing domain was meshed into 386,000 units. For the third set (fine grid), the computing domain was also meshed into 386,000 units, but the increasing grid ratio decreased from 1.03 to 1.015 , i.e. the grids became denser near the nozzle outlet. For the fourth set, the computing domain was meshed into 706,000 units; there is no obvious difference in pressure 
distribution, although the grids of the fourth set are denser than that of the third set. Therefore, the third grid set is independent enough for the study object, and was adopted for this research.

\subsection{Governing equations}

The CFD is rooted in the governing equations of fluid dynamics. These equations are the mathematical expressions of the following physical conversation laws:

(1) The mass conservation law of compressible fluid:

$\frac{\partial \rho}{\partial t}+\frac{\partial}{\partial x_{i}}\left(\rho u_{i}\right)=0$

(2) The momentum balance for the fluid in the computing domain:

$\frac{\partial}{\partial t}\left(\rho u_{i}\right)+\frac{\partial}{\partial x_{j}}\left(\rho u_{i} u_{j}\right)=-\frac{\partial p}{\partial x_{i}}+\frac{\partial}{\partial x_{j}}\left[\mu_{e f f}\left(\frac{\partial u_{i}}{\partial x_{j}}+\frac{\partial u_{j}}{\partial x_{i}}-\frac{2}{3} \delta_{i j} \frac{\partial u_{l}}{\partial x_{l}}\right)\right]+\frac{\partial}{\partial x_{j}}\left(-\rho \overline{\overline{u_{i}^{\prime} u_{j}^{\prime}}}\right)$

(3) The energy equation:

$\frac{\partial}{\partial t}(\rho E)+\frac{\partial}{\partial x_{i}}\left(u_{i}(\rho E+p)\right)=\frac{\partial}{\partial x_{i}}\left[\left(\alpha+\frac{c_{p} \mu_{t}}{p_{r t}}\right) \frac{\partial T}{\partial x_{i}}+u_{j}\left(\tau_{i j}\right)_{e f f}\right]$

where $\mathrm{E}$ and $\mathrm{T}$ are energy and temperature, respectively; $\tau_{i j}$ is the stress tensor:

$\tau_{i j}=\left[\mu\left(\frac{\partial u_{i}}{\partial x_{j}}+\frac{\partial u_{j}}{\partial x_{i}}\right)\right]-\frac{2}{3} \mu \frac{\partial u_{l}}{\partial x_{l}} \delta_{i j}$

where $\delta_{i j}$ is the viscous heat resulted from dissipation.

The equation of state for ideal gas was also introduced to close the system:

$p=\rho R T$

The turbulence models here rely on the Boussinesq eddy viscosity assumption, which links up Reynolds stress with mean velocity gradient. In other words, the Reynolds stress tensor obtained from the averaging operation is proportional to the mean deformation rate tensor:

$-\rho \overline{u_{i}^{\prime} u_{j}^{\prime}}=\mu\left(\frac{\partial u_{i}}{\partial x_{j}}+\frac{\partial u_{j}}{\partial x_{i}}\right)-\frac{2}{3}\left(\rho k+\mu_{t} \frac{\partial u_{i}}{\partial x_{i}}\right) \delta_{i j}$

The simplest complete turbulence model is the standard two-equation model. In such a model, the two separate transport equations can yield the turbulent velocity and turbulent length, respectively. Proposed by Launder and Spalding, the standard k- $\varepsilon$ model is a popular two-equation model for simulating industrial flow and heat transfer in engineering practices. The popularity is attributable to its robustness, economy and accuracy under various turbulent flows $[21,28]$.

The turbulence kinetic energy $(\mathrm{k})$ and its rate of dissipation ( $\varepsilon$ can be expressed as:

$\frac{\partial(\rho k)}{\partial t}+\frac{\partial}{\partial x_{i}}\left(\rho k u_{i}\right)=\frac{\partial}{\partial x_{j}}\left[\left(\mu+\frac{\mu_{t}}{\sigma_{k}}\right) \frac{\partial k}{\partial x_{j}}\right]+G_{k}+G_{b}-\rho \varepsilon-Y_{M}$ $\frac{\partial(\rho \varepsilon)}{\partial t}+\frac{\partial}{\partial x_{i}}\left(\rho \varepsilon u_{i}\right)=\frac{\partial}{\partial x_{j}}\left[\left(\mu+\frac{\mu_{t}}{\sigma_{\varepsilon}}\right) \frac{\partial \varepsilon}{\partial x_{j}}\right]+C_{1 \varepsilon} \frac{\varepsilon}{k}\left(G_{k}+C_{3 \varepsilon} G_{b}\right)-C_{2 \varepsilon} \rho \frac{\varepsilon^{2}}{k}$

where $G_{k}$ is the generation of turbulence kinetic energy under mean velocity gradient; $G_{b}$ is the generation of turbulence kinetic energy under buoyancy; $\mathrm{Y}_{\mathrm{m}}$ is the contribution of the fluctuating dilatation in compressible turbulence to the overall dissipation rate; $\mathrm{C}_{\mathrm{z} 1}, \mathrm{C}_{\mathrm{z} 2}$ and $\mathrm{C}_{\mathrm{z} 3}$ are constants' $\sigma_{k}$ and $\sigma_{z}$ are the turbulent Prandtl numbers for $\mathrm{k}$ and $\varepsilon$, respectively.

\subsection{Numerical plan and solution convergence}

The $\mathrm{k}-\varepsilon$ turbulence model was applied to solve the Reynolds-averaged Navier-Stokes equations for compressible fluid by the finite volume method on the commercial CFD software FLUENT 6.3.

The nozzle inlet was simulated by the pressure inlet boundary conditions, that is, the nozzle inlet has the same pressure with the pulse gas tank. The inlet of the secondary gas flow and the outlet of the computing domain were modelled with the pressure boundary conditions (1bar), i.e. the two places are under the atmospheric pressure. Considering the symmetrical structure of the computing domain, only a quarter of the nozzle was modelled by the symmetry boundary conditions. The ideal gas approximation of dry air was adopted as the working fluid, and all walls were considered adiabatic and slip-free.

For all equations, the convective terms were discretized using a second-order upwind scheme. The implicit formulations were used for the sake of accuracy and stability.

\subsection{Verification of simulation model}

To validate the simulation model, two experiments were conducted without filters, respectively at the pulse durations of $0.8 \mathrm{~s}$ and $1.0 \mathrm{~s}$. The experimental mass flow rate is the quotient of the mass loss in the high-pressure pulse gas tank and the pulse duration.

Figure 3 compares the simulated mass flow rates with the experimental results at different pulse pressures. It can be seen that the mean mass flow rate was more accurate at the longer pulse duration. This is because the longer duration suppresses the impact of the start and end of pulse cleaning. Besides, the mass flow rate decreased with the extension of the pulse duration, for it is difficult to extend the duration without changing the pulse pressure. In general, the simulated mass flow rate agrees well with the experimental results, indicating that the simulation model is suitable for predicting the cleaning performance of the rectangular slot nozzle.

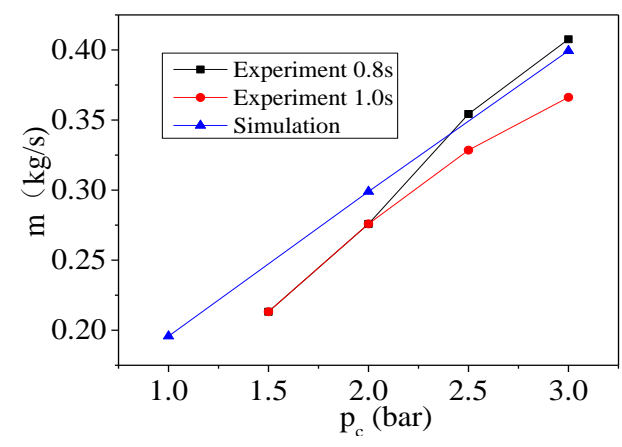

Figure 3. Model validation 


\section{RESULTS AND DISCUSSION}

\subsection{Flow field around the nozzle}

Let $\mathrm{x}, \mathrm{y}$ and $\mathrm{z}$ denote the vertical direction, contraction direction, and horizontal plane of the nozzle, respectively. The velocity profile along the $y$-direction at different positions along the $\mathrm{x}$-direction in plane $\mathrm{z}$ is illustrated in Figure 4. It can be seen that the velocity in the core zone of pulse jet cleaning was much faster than that in the surrounding zone. The effective distance along the y-direction was shorter than $50 \mathrm{~mm}$ from the centreline, and decreased gradually as the pulse gas moved away from the nozzle. The pulse gas spread faster than sound near the core zone, while the gas in the surrounding zone migrated at about $10 \mathrm{~m} / \mathrm{s}$. Viscous turbulence was observed in the core zone near the nozzle tip, such as the position of $\mathrm{x}=10 \mathrm{~mm}$, where the velocity was almost the same with that in the core zone. Within the core zone, the velocity was relatively slow on the centreline at the same position along the $\mathrm{x}$ direction, namely $x=30 \mathrm{~mm}$ and $\mathrm{x}=50 \mathrm{~mm}$. This phenomenon is caused by the shock wave. At $x=150 \mathrm{~mm}$, the viscous flow was fully developed from the pulse gas, and the velocity decreased gradually from the centreline to the surrounding positions.

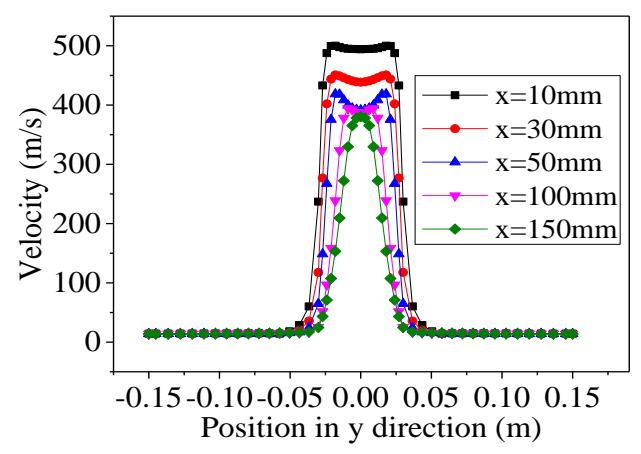

Figure 4. Variation of velocity along the y-direction

According to the pressure profile along the y-direction at different positions along the $\mathrm{x}$-direction (Figure 5), the pulse pressure in the core zone kept fluctuating around the surrounding pressure. As the result of the formation of the shock wave, the repeating cycles of positive and negative pulse pressures along the $\mathrm{x}$-direction reflect the induction and repulsion effects of the surrounding gas.

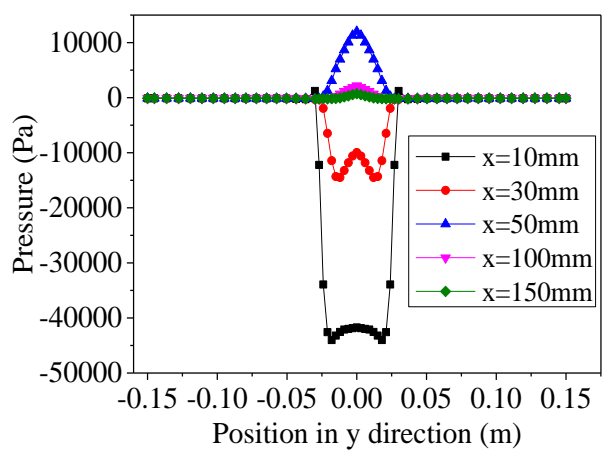

Figure 5. Variation of pressure along the y-direction

Figure 6 illustrates the pressure distribution along the nozzle axis at different pulse pressures. As shown in the figure, the pulse pressure has a major impact on the axial distribution of the pressure. In the straight segment $(\mathrm{x}=-400 \mathrm{~mm} \sim-210 \mathrm{~mm})$, the static pressure remained constant at the pulse pressure $\mathrm{p} 0$. In the convergence segment $(x=-210 \mathrm{~mm} \sim-150 \mathrm{~mm})$, the pressure dropped from the stagnation pressure $\mathrm{p} 0$ at the inlet, where the Mach number is small. In the divergence segment $(x=-150 \mathrm{~mm} \sim-600 \mathrm{~mm})$, the shock wave was generated in the flow field outside the nozzle outlet $[2,29]$. The pressure changed rapidly in the shock train area near the nozzle outlet, indicating that the pressure increase in the flow field relies heavily on the length of the shock train. The pressure recovered to the atmospheric pressure at the downstream distance greater than that length. It is observed that the pulse pressure is positively correlated with the shock-train length, the highest/lowest pressures in the shock train area, and the Mach number of the shock wave.

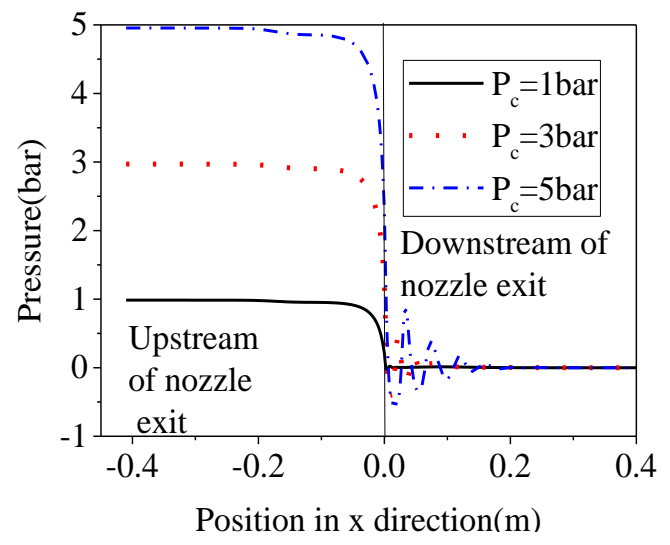

Figure 6. Pressure variation with $\mathrm{Pc}$ along $\mathrm{x}$ axis

Figure 7 describes the variation of the Mach number along the y-direction. It can be inferred from the figure that the Mach number increased from 0 to 1 in the nozzle, and multiple shocks appeared in the flow field at the nozzle outlet. Besides, the supersonic gas flow was decelerated through the shock system, and the velocity changed rapidly in the shock train region near the nozzle outlet. This means the pressure increase in the flow field relies heavily on the length of the shock train. The pressure also recovered to the atmospheric pressure at the downstream distance greater than that length. It is observed that the pulse pressure is positively correlated with the shocktrain length and the Mach number of the shock wave.

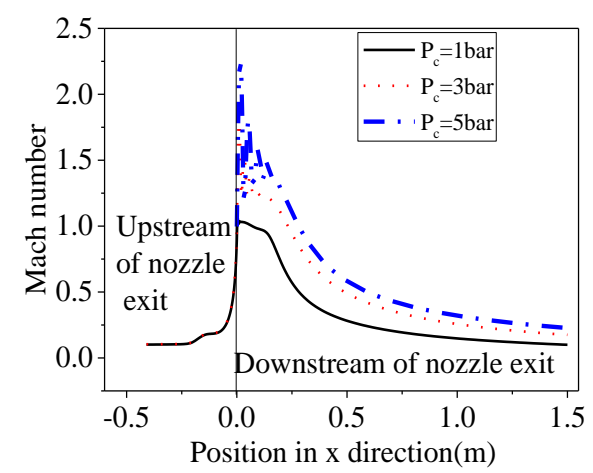

Figure 7. Much number variation with $\mathrm{Pc}$ along $\mathrm{x}$ axis

\subsection{Effect of nozzle configuration}

To disclose the effect of nozzle configuration on filtering performance, the primary mass and secondary mass were 
introduced to describe the mass flow rate of the gas entering through the nozzle and that of the gas entrained in the surrounding gas, respectively. Then, the sum of the two masses is denoted as the total mass, and the entrainment ratio $\left(\mathrm{R}_{\mathrm{en}}\right)$ was defined as below:

$\mathrm{R}_{\mathrm{e} n}=\frac{m_{e}}{m_{n}}=\frac{m_{t}-m_{n}}{m_{n}}$

Figure 8 illustrates how the primary mass, secondary mass, total mass and entrainment ratio change with nozzle outlet heights at the pulse pressure of 3 bar. In general, the primary mass increased linearly with the increase of the nozzle outlet height. The secondary and total masses rose at a decreasing rate, as the nozzle outlet increased in height. Moreover, the entrainment ratio plunged deeply as the nozzle outlet height grew from $1 \mathrm{~mm}$ to $7 \mathrm{~mm}$; the decline became gentle with further increase in the latter.

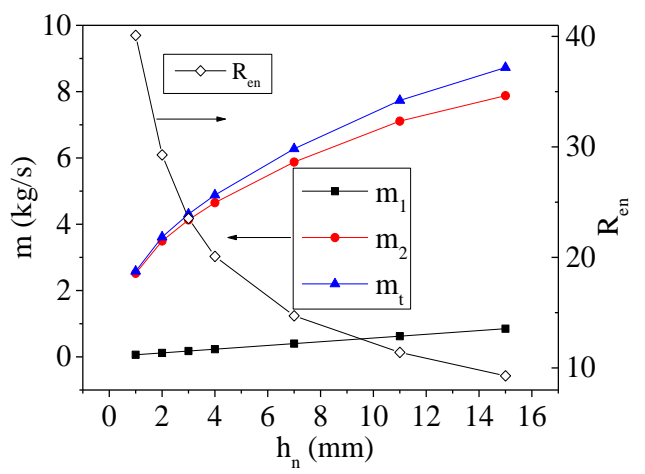

Figure 8. Effect of $h_{n}$ on cleaning efficiency

Figure 9 displays the variation of the primary mass, secondary mass, total mass and entrainment ratio with nozzle outlet widths at the pulse pressure of 3 bar. It can be seen that the primary, secondary and total masses all increased with the nozzle outlet width, while the entrainment ratio exhibited a gradual decrease as the nozzle outlet expanded in width.

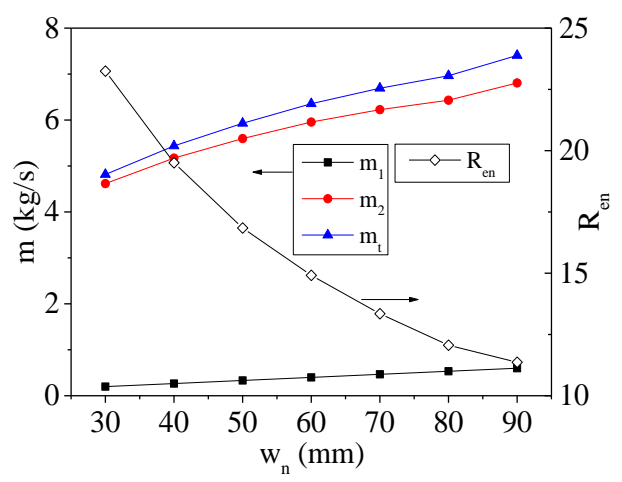

Figure 9. Effect of wn on cleaning efficiency

Figure 10 describes the relationship between the primary mass, secondary mass, total mass and entrainment ratio and nozzle extension width at the pulse pressure of 3 bar. As can be seen from the figure, the primary mass remained constant although the nozzle extension width increased from $60 \mathrm{~mm}$ to $110 \mathrm{~mm}$. The secondary and total masses underwent a gradual increase with the growth in the nozzle extension width. The entrainment ratio experienced a slight increase as the nozzle extension width expanded from $60 \mathrm{~mm}$ to $80 \mathrm{~mm}$, and then a sharp rise as the latter grew from $80 \mathrm{~mm}$ to $110 \mathrm{~mm}$.

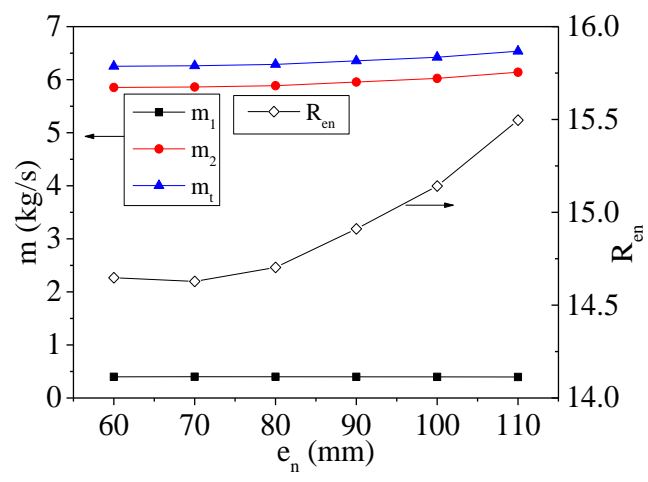

Figure 10. Effect of $e_{n}$ on cleaning efficiency

\subsection{Effect of diffuser configuration}

To discuss the effect of diffuser configuration on filtering performance, the proposed model was adopted for a simulation at different heights $\left(\mathrm{h}_{\mathrm{d}}\right)$, inlet widths $\left(\mathrm{w}_{\mathrm{d}}\right)$, and inlet-throat distances $\left(\mathrm{L}_{\mathrm{dt}}\right)$ of the diffuser. During the simulation, the nozzle configuration was set to a constant value, depending on the results of previous simulations. In addition, the nozzle outlet height $\left(\mathrm{h}_{\mathrm{n}}\right)$, nozzle outlet width $\left(\mathrm{w}_{\mathrm{n}}\right)$, and nozzle extension width $\left(e_{n}\right)$ were set to $7 \mathrm{~mm}, 60 \mathrm{~mm}$ and $90 \mathrm{~mm}$, respectively.

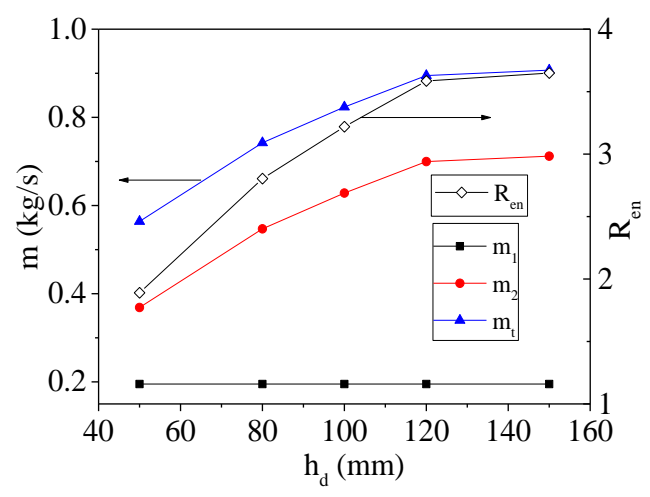

Figure 11. Effect of $h_{d}$ on cleaning efficiency

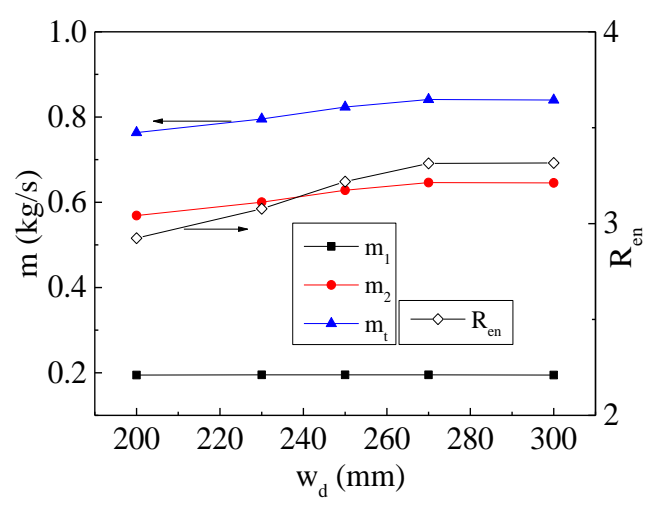

Figure 12. Effect of $w_{d}$ on cleaning efficiency

Figure 11 depicts the effect of diffuser height on pulse jet cleaning performance. It can be seen that the diffuser height 
almost had no impact on the primary mass, while the secondary mass, total mass and entrainment ratio increased with the diffuser height.

Figure 12 showcases the effect of diffuser inlet width on pulse jet cleaning performance. Obviously, the primary mass remained the same despite the increase of diffuser inlet width. By contrast, the secondary mass, total mass and entrainment ratio are positively correlated with diffuser inlet width.

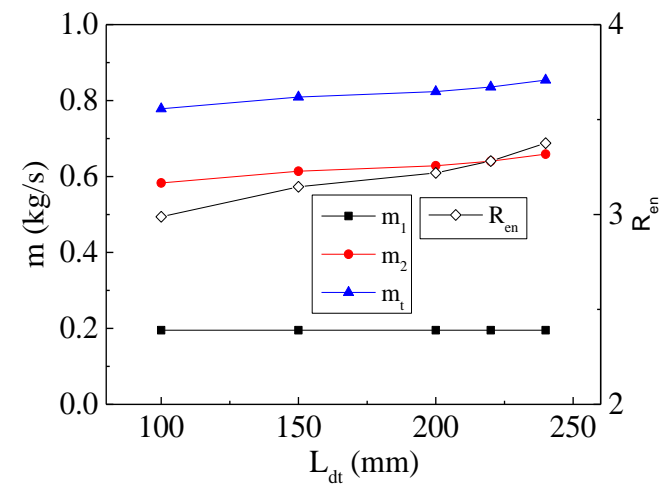

Figure 13. Effect of $\mathrm{L}_{d}$ on cleaning efficiency

Figure 13 illustrates the variation of the primary mass, secondary mass, total mass and entrainment ratio with inletthroat distances of the diffuser. The figure shows that, with the increase of the inlet-throat distance, the primary mass was basically unchanged, while the secondary mass, total mass and the entrainment ratio were on the rise. Comparing Figure 13 with Figures 11 and 12, the author concluded that diffuser height has a greater impact on the pulse jet cleaning performance than the other dimension parameters of the diffuser.

\subsection{Effect of nozzle-diffuser distance}

Without changing the nozzle and diffuser configurations, a series of simulation tests were performed at different distances between the nozzle and the diffuser. According to the test results in Figrue14, the nozzle-diffuser distance had nothing to do with the primary mass, while the second mass, total mass and entrainment ratio were positively correlated with that distance.

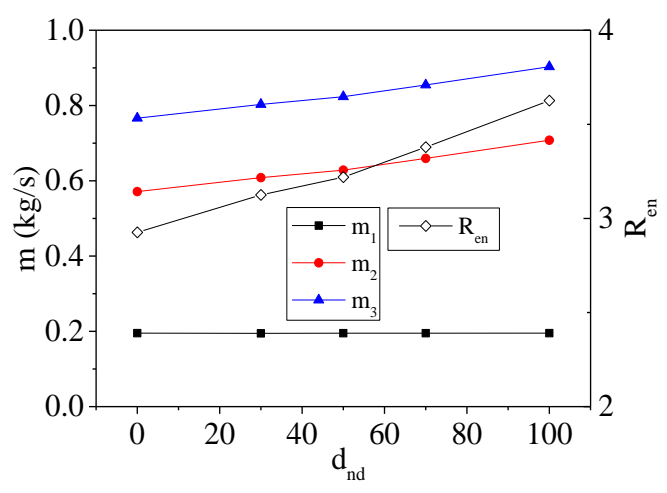

Figure 14. Effect of distance between the nozzle and diffuser on mass flow rate and entrainment ratio

\section{CONCLUSIONS}

This paper probes deep into the flow dynamics of rectangular slot nozzle through numerous CFD simulations. The main purpose is to investigate how the nozzle configuration, diffuser configuration and nozzle-diffuser distance influence the performance of pulse jet cleaning. The simulated results were verified against the data measured in the experiments. Finally, the author drew the following conclusions from the research:

First, obvious shocking waves were observed near the nozzle outlet. Second, the nozzle outlet height has a greater impact on pulse jet cleaning than other nozzle configuration parameters (e.g. nozzle outlet width and nozzle extension width), while the diffuser height has a greater impact on pulse jet cleaning than other diffuser configuration parameters (e.g. diffuser inlet width and inlet-throat distance). Third, the primary, secondary and total masses increased with the height and width of nozzle outlet; as the nozzle extension width grew, the secondary and total masses also increased, but the primary mass stayed the same. Fourth, the entrainment ratio is negatively correlated with the height and width of nozzle outlet, and positively with nozzle extension width. Fifth, the primary mass had nothing to do with the diffuser configuration, while the secondary mass, the total mass and the entrainment ratio increased with the diffuser height, the diffuser width and the nozzle-diffuser distance.

\section{ACKNOWLEDGMENT}

The authors acknowledge the funding support of the national natural science foundation of china (No. U1504217).

\section{REFERENCES}

[1] Vehlow J. (2014). Air pollution control systems in WtE units: An overview. Waste Management 5: 1-17. https://doi.org/ 10.1016/j.wasmann.2014.05.025

[2] Choi JH, Seo YG, Chung JW. (2001). Experimental study on the nozzle effect of the pulse cleaning for the ceramic filter candle. Powder Technology 114: 129-135.

[3] Dittler A, Ferer MV, Mathur P, Djuranovic P. (2002). Patchy cleaning of rigid gas filters $\mid$ transient regeneration phenomena comparison of modeling to experiment. Powder Technology 124: 55-66.

[4] Hashaikeh R, Lalia BS, Kochkodan V, Hilal N. (2014). A novel in situ membrane cleaning method using periodic electrolysis. Journal of Membrane Science 471: 149-154. https://doi.org/ 10.1016/j.memsci.2014.08.017

[5] Li HX, Li B, Bai X. (2015). Three dimensional modeling of gas-solid coupled free and Porous flow in a filtration process. International Journal of Heat and Technology 33: 101-106.

[6] Sharma SD, Mclennan K, Dolan M, Nguyen T, Chase D. (2013). Design and performance evaluation of dry cleaning process for syngas. Fuel 108: 42-53. https://doi.org/ 10.1016/j.fuel.2011.02.041

[7] Morris WJ. (1984). Cleaning mechanisms in pulse jet fabric filters. Filtration and Separation 21(1): 50-54.

[8] Theodore I, Reynolds I, Corvini A, Buonicore A. (1975). Particulate control by pulsed-air bag-house filtration: describing equations and solutions. Proceedings of 2 nd 
Speciality on the User and Fabric Filtration Equipment, Buffalo ed.: 90.

[9] Choi JH, Sakong KM, Chi HC, Ji ZL. (2008).Aspects of nozzle effect on the pulse jet cleaning of a ceramic filter. 10th World Filtration Congress, April 14-18, Leipzig, German 3: 378-382.

[10] Subramanian G, Natarajan SK, Adhimoulame K, Natarajan K. (2014). Comparison of numerical and experimental investigations of jet ejector with blower. International Journal of Thermal Sciences 84: 134-142. https://doi.org/ 10.1016/j.ijthermalsci.2014.05.008

[11] Lo LM, Chen DR, Pui DYH. (2010). Experimental study of pleated fabric cartridges in a pulse-jet cleaned dust collector. Powder Technology 197: 141-149. https://doi.org/ 0.1016/j.powtec.2009.09.007

[12] Weidemann C, Vogt S, Nirschl H. (2014). Cleaning of filter media by pulsed flow - Establishment of dimensionless operation numbers describing the cleaning result. Journal of Food Engineering 132: 29-38. https://doi.org/ 10.1016/j.jfoodeng.2014.02.005

[13] Yan CP, Liu GJ, Chen HY. (2013). Effect of induced airflow on the surface static pressure of pleated fabric filter cartridges during pulse jet cleaning. Powder Technology 249: 424-430. https://doi.org/ 10.1016/j.powtec.2013.09.017

[14] Qian YL, Bi YX, Zhang Q, Chen HY. (2014). The optimized relationship between jet distance and nozzle diameter of a pulse-jet cartridge filter. Powder Technology 266: 191-195. https://doi.org/ 10.1016/j.powtec.2014.06.004

[15] Berbner S, Löffler F. (1993). Pulse jet cleaning of rigid filter elements at high temperature. In: Proceedings of 2nd International Symposium on Gas Cleaning at High Temperatures, Chapman \& Hall: 225-243

[16] Stöcklmayer CH, Höflinger W. (1998). Simulation of long-term behavior of regenerateable dust filters. Filtration and Separation 5: 373-377

[17] Stöcklmayer CH, Höflinger W. (1998). Simulation of the regeneration of dust filters. Mathematics and Computers in Simulation 46: 601-609.

[18] Zhang X, Chen HH, Ji ZL. (2009). Characterization of flow field in the exit of pulse Jet Nozzle for Hightemperature Filtrating. Chinese Journal of Mechanical Engineering 45(10): 96-100.

[19] Lo LM, Chen DR, Pui DYH. (2010). Experimental study of pleated fabric cartridges in a pulse-jet cleaned dust collector. Powder Technology 197: 141-149. https://doi.org/ 10.1016/j.powtec.2009.09.007

[20] Duo W, Kirkby NF, Seville JPK, Clift R. (1997). Patchy cleaning of rigid gas filters-I. A probabilistic model. Chemical Engineering Science 52(1): 141-151.

[21] Ramy H. (2013).A simplified method for modeling of round and square ceiling diffusers, Energy Buildings 64: 473-482. https://doi: 10.1016/j.enbuild.2013.05.021

[22] Durst SM. (1994). Advatage of an integration system for hot gas filtration using rigid ceramic elements. Filtrion and Separation 6: 25.

[23] Gregg W, Ridge B, Vendetti R, Hiawatha L, Lindsay R. (1995). Low pressure pulse jet dust collector. US Patent 421(5): 845 .

[24] Bounaouara H, Ettouati H, Ticha HB, Mhimid A, Sautet JC. (2015). Numerical simulation of gas-particles two phase flow in pipe of complex geometry: Pneumatic conveying of olive cake particles toward a dust burner. International Journal of Heat and Technology 33: 99-106.

[25] Chen N. (2015). Impact of vertical wind deflectors installed at parapet of residential balcony on indoor air flow and temperature. Oxidation Communications 38(2): 923-935.

[26] Hao PF, Gao JZ, Si WJ, Zhang P. (2015). Research on flow pattern of nitrogen tetroxide liquid in the valve during start and shutdown operation. Oxidation Communications 38(2): 994-1000.

[27] Shuai Li, Dawei Liu, Qiang Li. (2015).The optimal design of a wind tunnel model sting system based on the CFD method. International Journal of Heat and Technology 33: 137-144.

[28] Yi Q.J, Tian MC, Fang D. (2015). CFD simulation of airsteam condensation on an isothermal vertical plate. International Journal of Heat and Technology 33: 26-32.

\section{NOMENCLATURE}

$C_{\varepsilon 1} \rightarrow$ Constant

$C_{\varepsilon 2} \rightarrow$ Constant

$C_{\varepsilon 3} \rightarrow$ Constant

$\mathrm{e} \rightarrow$ Nozzle extension width $(\mathrm{mm})$

$\mathrm{E} \rightarrow$ Energy

Ren $\rightarrow$ Entrainment ratio

$G_{k} \rightarrow$ Generation of turbulence kinetic energy due to the mean velocity gradient

$G_{b} \rightarrow$ Generation of turbulence kinetic energy due to buoyancy $\mathrm{dnd} \rightarrow$ Distance between nozzle and diffuser $(\mathrm{mm})$

$\mathrm{hn} \rightarrow$ Nozzle outlet height $(\mathrm{mm})$

hd $\rightarrow$ Diffuser height $(\mathrm{mm})$

$\mathrm{wn} \rightarrow$ Width of nozzle $(\mathrm{mm})$

wd $\rightarrow$ Width of diffuser $(\mathrm{mm})$

en $\rightarrow$ extension width of nozzle $(\mathrm{mm})$

Ldt $\rightarrow$ Length of between the diffuser inlet and the diffuser throat (mm)

$\mathrm{i}, \mathrm{j} \rightarrow$ Unit vectors

$k \rightarrow$ Turbulent kinetic energy

$\mathrm{m} 1 \rightarrow$ Mass flow rate of gas at nozzle outlet $(\mathrm{kg} / \mathrm{s})$

$\mathrm{m} 2 \rightarrow$ Mass flow rate of entrained gas $(\mathrm{kg} / \mathrm{s})$

$\mathrm{mt} \rightarrow$ Mass flow rate of gas at nozzle downstream $(\mathrm{kg} / \mathrm{s})$

$\mathrm{pc} \rightarrow$ Pulse cleaning pressure(bar)

pint $\rightarrow$ Pressure at center line integrated with $\mathrm{x}$ axis $(\mathrm{Pa})$

$\mathrm{R} \rightarrow$ Characteristic gas constant $(\mathrm{J} / \mathrm{kg} . \mathrm{K})$

$\mathrm{T} \rightarrow$ Temperature $(\mathrm{K})$

$\mathrm{t} \rightarrow$ Time(s)

$\mathrm{u} \rightarrow \operatorname{Velocity}(\mathrm{m} / \mathrm{s})$

$Y_{M} \rightarrow$ Contribution of the fluctuating dilatation to the overall dissipation rate

\section{Greek letter}

$\delta \mathrm{ij} \rightarrow$ Kronecker symbol

$\rho \rightarrow \operatorname{density}(\mathrm{kg} /)$

$\tau \mathrm{ij} \rightarrow$ stress tensor

$\mu \mathrm{t} \rightarrow$ Turbulent viscosity

$\overline{u_{\imath}^{\prime} u_{\jmath}^{\prime}} \rightarrow$ Reynolds-stress tensor

$\varepsilon \rightarrow$ Rate of dissipation

$\sigma_{k} \rightarrow$ Turbulent Prandtl numbers for $\mathrm{k}$

$\sigma_{\varepsilon} \rightarrow$ Turbulent Prandtl numbers for $\varepsilon$ 